\title{
Связь релаксации собственного стимулированного пикосекундного излучения GaAs c характерным временем остывания носителей заряда
}

\author{
(C) Н.Н. Агеева, И.Л. Броневой , Д.Н. Забегаев, А.Н. Кривоносов \\ Институт радиотехники и электроники им. В.А. Котельникова Российской академии наук, \\ 125009 Москва, Россия \\ ฯE-mail: bil@cplire.ru
}

Поступила в Редакцию 18 марта 2019 г.

В окончательной редакции 9 апреля 2019 г.

Принята к публикации 23 мая 2019 г.

Во время мощной пикосекундной оптической накачки тонкого ( 1 мкм) слоя GaAs в нем возникает интенсивное (до 1 ГВт $/ \mathrm{cm}^{2}$ ) стимулированное пикосекундное излучение. Обнаружено, что при фиксированной плотности энергии импульса накачки с увеличением его диаметра растет характерное пикосекундное время релаксации излучения и плотности носителей $\tau_{r}$. Вследствие взаимосвязи плотности и температуры носителей при высокоинтенсивном излучении (в состоянии насыщения усиления излучения) $\tau_{r}$ связано с характерным временем релаксации температуры фотонакачанных носителей $\tau_{T}$, определенным ранее теоретически с учетом разогрева носителей излучением. Следующее из этого аналитическое выражение для $\tau_{r}$ как функции $\tau_{T}$ согласуется с вышеуказанными экспериментальными результатами.

Ключевые слова: релаксация стимулированного излучения, пикосекундное, остывание носителей заряда.

DOI: $10.21883 /$ FTP.2019.11.48442.9105

\section{1. Введение}

Собственное стимулированное (усиленное спонтанное) излучение, возникающее в тонком слое GaAs во время его оптической пикосекундной накачки, представляет (как в чисто научном, так и в прикладном аспектах) важный и интересный объект исследований. Для краткости будем называть его далее излучение. Помимо пикосекундного времени образования и релаксации излучения, высокой интенсивности, самыми основными его особенностями являются следующие. Излучение поддерживает электронно-дырочную плазму (ЭДП) в состоянии, в котором плотность и температура ЭДП взаимосвязаны. Стимулированный излучением энергетический транспорт носителей заряда вызывает замедление залечивания отклонений от квазиравновесного фермиевского распределения носителей заряда. Иными словами, энергетический транспорт замедляет восстановление фермиевского распределения. Спектральные моды излучения активны по отношению к вынужденному комбинационному рассеянию. Возникают пикосекундные автомодуляции излучения и фундаментального поглощения зондирующего пикосекундного импульса света в слое GaAs. Эти автомодуляции интерпретируются как следствие межзонных осцилляций электронов, возбуждаемых полем излучения и синхронизуемых вынужденным комбинационным рассеянием его спектральных мод. Следствием перечисленных свойств излучения является образование значительного числа разнообразных нелинейных пикосекундных оптоэлек- тронных эффектов ${ }^{1}$. Перейдем к работам, послужившим предпосылкой для обнаружения нового свойства стимулированного рекомбинационного излучения, которому посвящена настоящая статья.

В работе [1] исследовалась пикосекундная релаксация просветления (увеличения прозрачности), возникавшего при пикосекундной оптической накачке слоя GaAs. Измеренная корреляционным методом pump-probe, она отображала релаксацию заселенности носителями заряда энергетических уровней, располагавшихся в зонах проводимости и валентной и связанных прямым оптическим переходом. Характерное время той релаксации $\tau_{b}$ возрастало при увеличении диаметра луча накачки $D$, производимого с сохранением плотности энергии накачки $W_{\mathrm{ex}} / S=\mathrm{const}$, где $W_{\mathrm{ex}}-$ энергия импульса накачки, $S$ - площадь сечения луча накачки. Предположительное объяснение возрастания $\tau_{b}$ с $D$ базировалось на: $(a)$ взаимосвязи плотности и температуры ЭДП при мощном излучении, $(b)$ функциональной зависимости характерного времени остывания ЭДП в присутствии стимулированного излучения, теоретически определенной в [2].

В работе [3] при одном фиксированном диаметре $D$ было измерено в реальном времени излучение и получены некоторые его характеристики. В частности, было обнаружено, что излучение релаксирует с характерным временем $\tau_{r} \sim 10$ пс, совпадающим с его оценкой, кото-

\footnotetext{
${ }^{1}$ Интересующийся читатель может ознакомиться с их перечнем и списком основных публикаций, включающим публикации о вышеназванных свойствах излучения, на сайте ИРЭ им. В.А. Котельникова PAH http://www.cplire.ru/rus/bil/index.html
} 
рая тоже базировалась на положениях, названных выше в пунктах $(a)$ и $(b)$.

В настоящей работе при фиксированной плотности энергии накачки измерены зависимости от диаметра $D$ : $(c)$ спектра излучения; $(d)$ характерного времени релаксации излучения. Первая зависимость позволила определить ширину запрещенной зоны, плотность и температуру неравновесных носителей заряда в функции от диаметра $D$. Данные $(c)$ и $(d)$ позволили выявить связь релаксации излучения с характерным временем остывания носителей заряда, экспериментально подтвердив аналитическое выражение характерного времени релаксации интенсивности излучения и носителей заряда, основанное на этой связи.

\section{2. Эксперимент}

Опыты проводились при комнатной температуре $T_{R}$ на пикосекундном лазерном спектрофотохронометрическом комплексе с автоматической измерительной системой.

Комплекс состоит из: задающего YAG-лазера с диодной накачкой PL PDP1-300; системы усилителей лазерного импульса, накачиваемых источниками питания серии 700ТC; удвоителей частоты светового импульса (кристаллов KDP); двух параметрических генераторов света на $\mathrm{LiNbO}_{3}$ с температурной перестройкой длины волны - один для генерации накачивающего, второй - зондирующего или др. импульсов длительностью $(\mathrm{FWHM}) \approx 10$ пс с линейной поляризацией света; спектрофотохронометрической системы. Наиболее важными компонентами последней являлись: (a) двойной спектрограф SpectraPro-2500i, используемый как для спектральных измерений, так и (в режиме вычитания дисперсии) в качестве полосового фильтра длин волн, не искажающего длительность излучения; $(b)$ пикосекундная электронно-оптическая камера (ЭОК) PS-1/S1; (c) ПЗС-камеры PIXIS и CoolSNAP:HQ2 для регистрации соответственно спектра излучения и изменения со временем интенсивности спектральной компоненты излучения, пропущенной спектрографом (ПЗС - прибор с зарядовой связью).

Исследуемый образец представлял собой гетероструктуру $\mathrm{Al}_{0.32} \mathrm{Ga}_{0.68} \mathrm{As}-\mathrm{GaAs}-\mathrm{Al}_{0.32} \mathrm{Ga}_{0.68} \mathrm{As}$ с толщиной слоев соответственно 1.2-1.6-1.3 мкм. Слои $\mathrm{Al}_{x} \mathrm{Ga}_{1-x} \mathrm{As}$ прозрачны для света с $\hbar \omega<1.7$ эВ, используемого в экспериментах. На поверхности гетероструктуры (исключая поверхность торцов) было нанесено антиотражающее покрытие, гарантировавшее, в частности, отсутствие вертикального резонатора.

При межзонном поглощении мощного возбуждающего (ех) импульса света (накачки) с энергией фотона $\hbar \omega_{\mathrm{ex}}=1.558$ эВ, падавшего на образец под углом $10^{\circ}$ относительно нормали к его поверхности, в слое GaAs генерировалась ЭДП. Ее плотность была достаточной для возникновения в GaAs излучения. Излучение усиливалось преимущественно в направлении вдоль эпитаксиального слоя GaAs, что подтверждалось, помимо прочего, диаграммой направленности излучения, выходившего из образца [4]. Из-за несовершенства волноводных качеств гетероструктуры часть излучения вытекала через ее поверхность. Доля этой части излучения, распространявшаяся (если при оценке пренебречь диаметром активной области) внутри телесного угла $\Omega \approx 1.2 \cdot 10^{-2}$ стерадиан с осью, ортогональной поверхности гетероструктуры, фокусировалась на входную щель двойного спектрографа. Выбор именно этой части излучения для измерений обусловлен тем, что она выходит прямо из активной области. Таким образом она не изменяется из-за поглощения в пассивной области и отражения от торцов образца.

То, что излучение (и выходящее из торца образца, и вытекающее через поверхность гетероструктуры изза ее несовершенства) одно и то же стимулированное, доказано в наших предыдущих исследованиях излучения. Они были выполнены при тех же условиях фотонакачки и обнаружили все характерные свойства стимулированного излучения, перечисленные далее.

- Наличие в спектре фундаментального поглощения света области усиления света $[4,5]$.

- Появление излучения при энергии накачки, превышающей некоторое пороговое значение [6].

- Появление излучения при плотности неравновесных носителей заряда $n=p>n_{\text {th }}$, где $n_{\text {th }} \approx$ $\approx 1.3 \cdot 10^{18} \mathrm{~cm}^{-3}-$ пороговая плотность носителей при комнатной температуре [7].

- Узкая диаграмма направленности излучения, при которой основная часть излучения выходит из торцов образца [4]. Значительно меньшая часть вытекает через поверхности гетероструктуры, параллельные эпитаксиальным слоям. Это происходит вследствие несовершенства волноводных свойств гетероструктуры [4,8]. Возможными причинами такого вытекания излучения могут быть: рэлеевское рассеяние, рассеяния Ми, френелевское отражение (это зависит от размера дефектов).

- Имеется еще следующее важное доказательство того, что излучение, вытекающее через поверхность гетероструктуры, является частью стимулированного излучения, распространяющегося вдоль эпитаксиального слоя. Обнаруженная в работе [9] модуляционная компонента спектра части излучения, вытекавшей через поверхность гетероструктуры по нормали к ней, представляла собой ряд эквидистантно расположенных локальных максимумов (см. рис. 2 в [10]). Спектральные компоненты излучения с энергиями фотона $\hbar \omega$, для которых наблюдались указанные максимумы, назовем $i$-модами. Интервал, которым приблизительно разделены $i$-моды, совпадает с расчетным интервалом между резонансными модами излучения, зигзагообразно распространяющегося внутри слоя GaAs, рассматриваемого как активный резонатор, под углом $\beta \approx 5.3^{\circ}$ к границам раздела слоев $\mathrm{Al}_{x} \mathrm{Ga}_{1-x} \mathrm{As}$ и GaAs. Распространение мод именно под 
таким углом следует и из диаграммы направленности излучения, измеренной в [4]. Согласно диаграмме, излучение максимальной интенсивности выходило из торца образца под углом $\varphi \approx 20^{\circ}$, как и должно выходить излучение, по закону Снеллиуса: $\chi \sin \beta=\sin \varphi$, где $\chi=3.6$ - показатель преломления GaAs.

- Спектр исследуемого излучения существенно уже спектра спонтанного излучения, причем сужается он в направлении к своему длинноволновому краю при увеличении энергии импульса накачки или диаметра луча накачки [11].

Очевидно, что и особенности излучения, перечисленные во Введении, могут быть присущи только стимулированному излучению.

Диаметр луча накачки (FWHM) в местоположении образца D последовательно изменяли от 0.2 до $1.1 \mathrm{Mм.}$ При этом, как уже говорилось, плотность накачки $W_{\mathrm{ex}} / S=4 W_{\mathrm{ex}} / \pi D^{2}$ поддерживалась неизменной. Измерение диаметра луча в местоположении образца предварительно проводилось с помощью веб-камеры. При каждом фиксированном $D$ временны́е формы (огибающие), т.е. хронограммы измерялись для следующих импульсов: интегрального по спектру излучения $H_{s}(t)$, накачки $H_{\mathrm{ex}}(t)$ и опорного $(p) H_{p}(t)$.

Взаимное распределение во времени импульсов накачки и излучения устанавливалось следующим образом. Параметрические генераторы света, в которых генерировались импульсы накачки и опорные, одновременно накачивались двумя частями одного и того же светового импульса. Задержки $\theta$ между импульсами накачки и опорными импульсами были измерены (прокалиброваны) ЭОК при всех используемых в экспериментах диаметрах луча накачки. Вместе с измеряемым импульсом излучения в спектрограф поступал с регулируемой задержкой опорный импульс с длиной волны, близкой к спектру излучения. Хронограммы излучения и опорного импульса измерялись одномоментно (рис. 1). Это позволяло определить расположение во времени излучения относительно опорного импульса и, учитывая затем $\theta$, относительно импульса накачки.

При измерении спектров и хронограмм излучения в расчет принимались только те акты накачки, для которых отклонения энергии импульса накачки от заданного значения не превышали $\pm 5 \%$. По результатам 160 измерений определялся усредненный спектр или хронограмма. Большее число измерений не требовалось, поскольку отклонения (изменения) усредненного спектра или хронограммы при дальнейших измерениях не превышали $2 \%$. При измерении хронограмм осуществлялась автоматическая компенсация джиттера (нестабильности запуска линейной развертки) ЭОК [12]. Сам же джиттер не превышал \pm 4.5 пс. Полученные хронограммы подвергались прецизионному сглаживанию, чтобы избежать влияния остатков шумов. Сглаживание проводилось по алгоритму быстрого преобразования Фурье с отсечением высокочастотных колебаний (FFT filter).

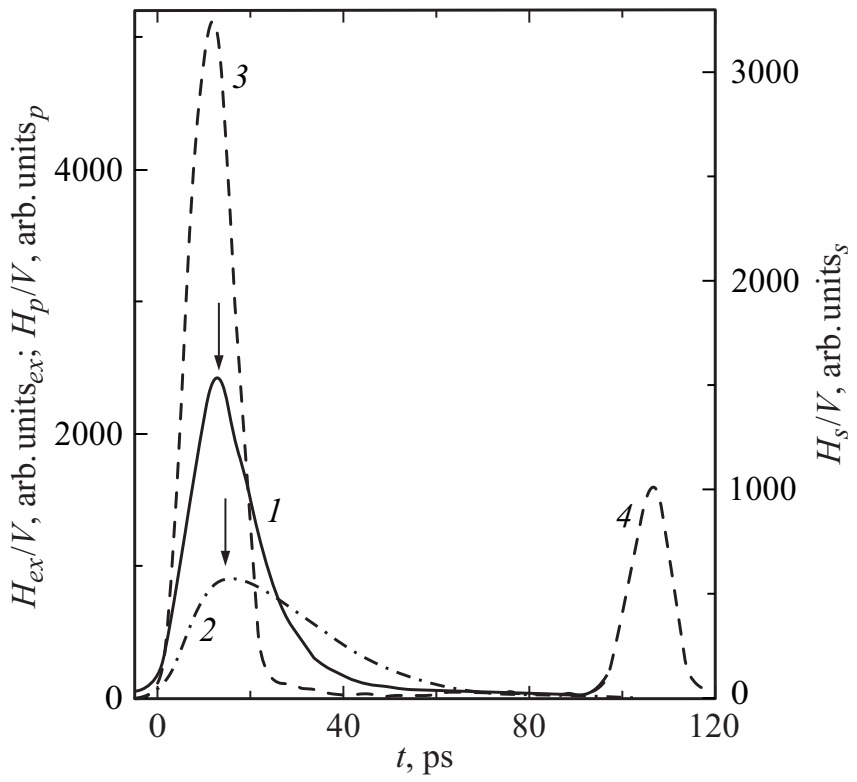

Рис. 1. Хронограммы излучения $H_{s}(t)$ при диаметрах луча накачки $D=0.21$ мм $(1)$ и $D=1.1$ мм (2), накачки $H_{\mathrm{ex}}(t)(3)$, нормированные на эффективный объем $V$ активной зоны. Хронограмма опорного импульса $H_{p}(t)(4)$. Стрелки указывают на максимум (пик) хронограмм $H_{s} / V=f(t)$, обозначаемый в тексте $H_{\text {max }}$.

На рис. 1 представлены в качестве примера хронограммы накачки и вызываемого ею при $D=0.21$ и 1.1 мм излучения. Хронограммы нормированы на эффективный объем активной зоны $V=d \pi D^{2} / 4$. Смысл нормировки поясняется в следующем разделе. Релаксацию излучения в некотором интервале времени, в котором оно ослабевает более чем на порядок, можно аппроксимировать как экспоненциальную с характерным временем $\tau_{r}$. Обнаруживается, что $\tau_{r}$ меняется с $D$. Это иллюстрирует рис. 2, где нормированные хронограммы излучения $H_{s}(t) / V$ при $D=0.21$ и 1.1 мм представлены в полулогарифмическом масштабе. Изменение времени $\tau_{r}$ при изменении диаметра $D$ в диапазоне от 0.2 до 1.1 мм представлено на рис. 3. Происходящее на большей части этого диапазона возрастание $\tau_{r}$ насыщается в конце диапазона.

Пример измеренного интегрального по времени спектра излучения дан на вставке к рис. 4 для $D=0.7$ мм.

\section{3. Обсуждение результатов}

Для всех наших экспериментов, выполненных в течение последних тридцати лет, общим было следующее. Мощной пикосекундной оптической накачке подвергался тонкий ( 1 мкм) слой GaAs, входивший в состав названной выше гетероструктуры. Этот материала важен для полупроводниковой оптоэлектроники и его параметры хорошо известны [13]. B GaAs генеририровалось 
высокоинтенсивное пикосекундное стимулированное излучение. Такая общность позволяет нам использовать физические зависимости, установленные в одних работах, при объяснении экспериментальных результатов, полученных в других работах и, в частности, в нижеследующем обсуждении. Допустимо предполагать, что, во-первых, в каждый момент времени вытекающая ортогонально эпитаксиальным слоям часть излучения пропорциональна, как и все излучение, скорости стимулированной излучательной рекомбинации неравновесных (накачанных) носителей заряда. Во-вторых, что последняя намного больше скорости других типов рекомбинации, которые поэтому можно не учитывать. Тогда измерявшуюся в эксперименте хронограмму излучения $H_{s}(t)$ связывает с плотностью ЭДП соотношение

$$
\frac{H_{s}}{V} \propto\left|\frac{d n}{d t}\right|,
$$

где $n$ - плотность электронов в зоне проводимости, равная плотности дырок в валентной зоне. Отсюда следует, что графики $H_{s} / V=f(t)$ на рис. 1 одновременно иллюстрируют относительное изменение со временем $t$ скорости стимулированной рекомбинации

$$
R_{s t}=\left|\frac{d n}{d t}\right|=f(t)
$$

для двух диаметров $D$.

В некотором интервале времени, с некоего момента, обозначим его $t_{0}$, наблюдается экспоненциальное уменьшение $H_{s}$ со временем $t$ (рис. 2). Это можно представить как

$$
\frac{H_{s}}{V}=\left[\frac{H_{s}}{V}\right]_{t=t_{0}} \exp \left(-\left(t-t_{0}\right) / \tau_{r}\right) .
$$

Соответственно и скорость стимулированной рекомбинации в том же интервале времени, учитывая (1), уменьшается с тем же характерным временем $\tau_{r}$

$$
\frac{d n}{d t}=\left[\frac{d n}{d t}\right]_{t=t_{0}} \exp \left(-\left(t-t_{0}\right) / \tau_{r}\right) .
$$

Решением уравнения (3) является следующее выражение для плотности носителей:

$$
n=\left(n_{t=t_{0}}-n_{\mathrm{th}}\right) \exp \left(-\left(t-t_{0}\right) / \tau_{r}\right)+n_{\mathrm{th}} .
$$

В (4) учтено, что когда плотность носителей спадает до $n_{\mathrm{th}}$, стимулированное излучение прекращается, и носители далее уже не рекомбинируют стимулированно.

Из (4) видно, что плотность ЭДП релаксирует с характерным временем $\tau_{r}$. Дифференцируя (4), получаем то же по сути выражение, что и (3), но в виде, общепринятом для рекомбинации:

$$
\left|\frac{d n}{d t}\right|=\frac{n-n_{\mathrm{th}}}{\tau_{r}} .
$$

Из (5) следует, что скорость стимулированной пикосекундной рекомбинации определяется отношением той

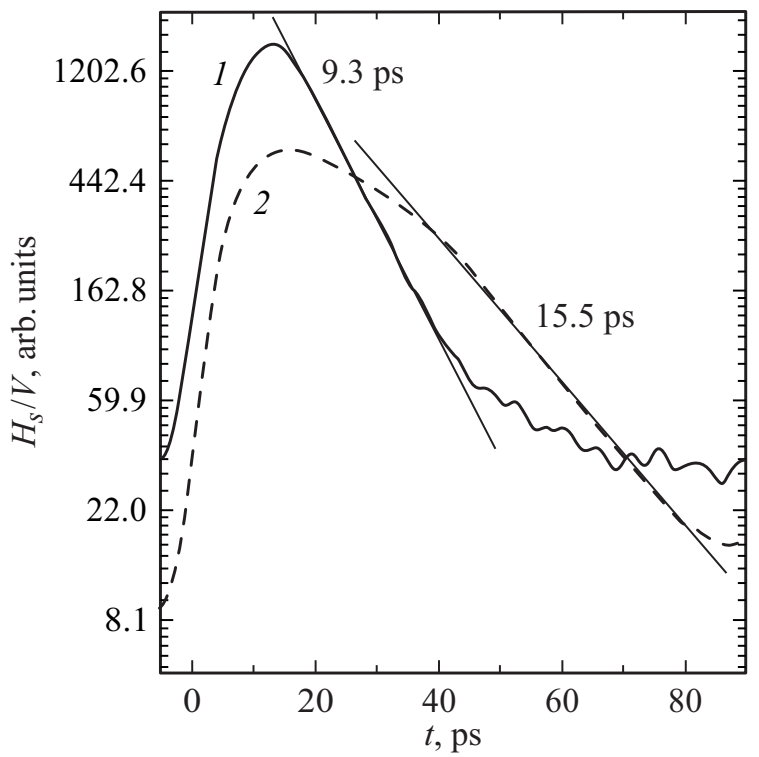

Рис. 2. Нормированные на объем $V$ хронограммы излучения для $D=0.21$ (1) $D=1.1$ мм (2), представленные в полулогарифмическом масштабе. К их спадам проведены касательные линии, показывающие, что в некотором интервале времени релаксацию излучения можно аппроксимировать как экспоненциальную с характерным временем $\tau_{r}$, указанным рядом с касательной.

надпороговой части плотности ЭДП $\Delta n=n-n_{\mathrm{th}}$, которая стимулированно рекомбинирует, к характерному времени ее релаксации $\tau_{r}$. Напомним, что последнее определяется экспериментально в настоящей работе как характерное время релаксации излучения.

Уточним, что спектральные компоненты излучения появляются не одновременно, а в некой последовательности, как обнаружено в [9]. В эксперименте время $t_{0}$ (меняющееся с $D)$ - это момент времени, когда длинноволновые компоненты излучения, появляющиеся последними, достигают максимума. Соответственно при $t>t_{0}$ спектральные компоненты излучения только спадают [3].

Для разных типов безызлучательной рекомбинации и спонтанной излучательной рекомбинации время $\tau_{r}$ в формуле, подобной (5), отличается определяющим его выражением [14]. Далее мы покажем, каким аналитическим выражением может определяться характерное время $\tau_{r}$ в (5) в случае пикосекундной излучательной рекомбинации, стимулированной излучением.

Изменение $\tau_{r}$ с $D$, иллюстрируемое рис. 3, можно интерпретировать с помощью следующего представления, использовавшегося в [3], и еще ранее при рассмотрении релаксации просветления фотонакачанного слоя GaAs [1].

Пока энергия фотона накачки $\hbar \omega_{\mathrm{ex}}$ ненамного превышает ширину запрещенной зоны $E_{g}$, то и температура генерируемой плотной ЭДП не сильно превышает приблизительно комнатную температуру решетки (например, в работе [5] при $\hbar \omega_{\mathrm{ex}}=1.52$ эВ температура 


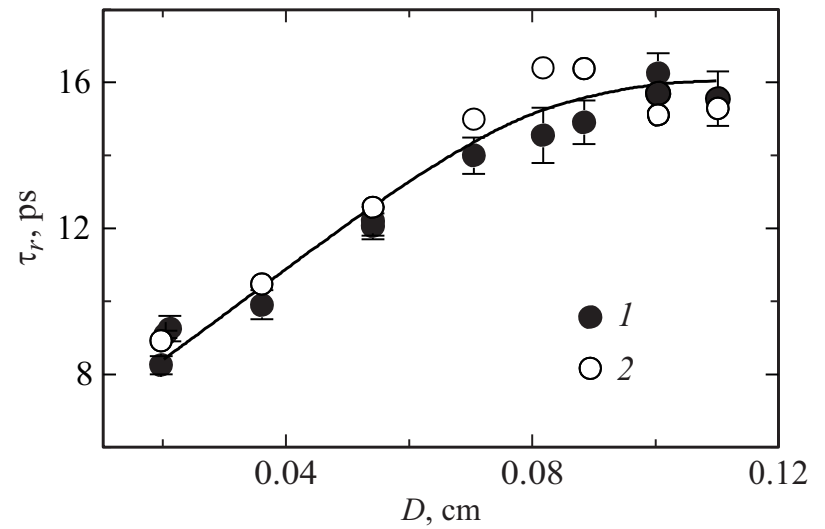

Рис. 3. Зависимость времени $\tau_{r}$ от диаметра $D .1$ - экспериментальные точки, 2 - расчет, сплошная кривая проведена для наглядности.

ЭДП $T_{c}$ не превышала $\left.470 \mathrm{~K}\right)$. Существенно возрастать инверсии заселенностей, возникающей при накачке, не дает, во-первых, интенсивное стимулированное рекомбинационное излучение $[4,5]$ (подобное принято называть еще насыщением усиления). Во-вторых, периодическая трансляция вверх по зоне проводимости локального обеднения заселенностей электронов, создаваемого излучением на дне зоны [4 и др.]. Период равен энергии продольного оптического фонона, и трансляция происходит для поддержания детального равновесия. Будем пренебрегать, во-первых, такой модуляцией энергетического распределения электронов, во-вторых, той малой долей плотности ЭДП, которая образует инверсию заселенностей. Будем приближенно считать распределение фермиевским. Тогда энергетическое распределение ЭДП можно характеризовать условиями $[5,15]$ :

$$
n=p, \quad \mu_{e}-\mu_{h} \approx E_{g},
$$

где $p$ - плотность дырок в валентной зоне, $\mu_{e}$ и $\mu_{h}$ - квазиуровни Ферми электронов и дырок соответственно. Такое приближение позволило ранее объяснить: обратимое пикосекундное просветление GaAs [15], распределение электронов между долинами, величину сужения запрещенной зоны GaAs из-за кулоновского взаимодействия носителей заряда, усиление излучения в результате стимулированного им рамановского рассеяния возбуждающего света на связанных плазмонфононных колебаниях [7]. В таком приближении плотность $n$ и температура $T_{c}$ ЭДП взаимосвязаны. Для рассматриваемого диапазона $T_{c}$ можно принять, что

$$
n-n_{\mathrm{th}}=k\left(T_{c}-T_{R}\right)^{3 / 2},
$$

где $k$ - коэффициент, не требующий здесь уточнения. Такой аппроксимации соответствует экспериментальная зависимость, составленная из результатов $[5,16]$, которая близка к приведенной в [7] расчетной зависимости, удовлетворявшей условиям (6), - это иллюстрирует рис. 6 в работе [3].
Из соотношений (4) и (7) элементарно математически выводится, что температура $T_{c}$ релаксирует экспоненциально с характерным временем $\tau_{T}$, которое связано с $\tau_{r}$ соотношением

$$
\tau_{r} \approx(2 / 3) \tau_{T} .
$$

В теории [2], разработанной практически для тех же условий, что и в анализируемом в данной статье эксперименте, было учтено следующее. Интенсивное стимулированное излучение приводит к дополнительному разогреву ЭДП, замедляющему ее охлаждение. В [2] было получено следующее аналитическое выражение для характерного времени релаксации температуры ЭДП в GaAs при наличии интенсивного собственного стимулированного излучения:

$$
\tau_{T} \approx\left(A+B \tau_{p} T_{c}^{1 / 2} E_{g}\right) \tau_{h} .
$$

Подставляя это выражение в (8), получаем аналитическое выражение для времени $\tau_{r}$, отличающееся от (9) только числовым коэффициентом:

$$
\tau_{r} \approx(2 / 3)\left(A+B \tau_{p} T_{c}^{1 / 2} E_{g}\right) \tau_{h} .
$$

Здесь $A=6.3, B=0.36 ; \tau_{h} \approx 0.8$ пс - время релаксации энергии ЭДП за счет эмиссии оптических фононов с учетом разогрева последних [17], $\tau_{r}, \tau_{p}, \tau_{h}$ измеряются в пс, $T_{c}-$ в K, $E_{g}-$ в эВ. Для лучшего согласия с экспериментом значения $A$ и $B$ незначительно изменены по сравнению с полученными в [2] $A=6.2$ и $B=0.34$. Первое слагаемое в выражении (10) учитывает разогрев ЭДП, связанный с тем, что энергия носителей заряда, участвующих в стимулированной рекомбинации, меньше средней энергии носителей в ЭДП. Второе слагаемое учитывает разогрев ЭДП из-за внутризонного поглощения собственного излучения. Заметим, что совместное влияние на $T_{c}$ и $n$ обоих механизмов разогрева наблюдалось, например, в [18], а только внутризонного поглощения света - в [19]. Время $\tau_{p}-$ это характерное время движения фотона излучения в активной среде, пока фотон не покинет эту среду или не будет поглощен:

$$
\tau_{p}^{-1} \approx c \chi^{-1}\left(\gamma+D^{-1}\right) .
$$

Здесь $\gamma=\sigma n-$ коэффициент внутризонного поглощения света, $\sigma=1.5 \cdot 10^{-17} \mathrm{~cm}^{2}$ [13]. Сделаем теперь на основании (10) оценку $\tau_{r}$.

Максимальную плотность ЭДП $n_{m}=p_{m}$, достигавшуюся в описанном эксперименте при каждом конкретном значении диаметра $D$, определяем с помощью расчетной зависимости $E_{g}=f(n)$, представленной сплошной линией на рис. 2 в работе [7]. Такая зависимость представляет перенормировку (сужение) ширины запрещенной зоны вследствие кулоновского взаимодействия носителей заряда [20-23] и экспериментально подтверждена в [7]. Указанная зависимость применима до тех пор, пока энергетический транспорт носителей заряда не возрастает настолько, что начинает возникать дефицит перенормировки [24]. В настоящей работе накачка была 
недостаточна для создания такого дефицита. Предполагалось, что при фиксированном $D$ предельно суженная ширина запрещенной зоны - это $E_{g-\min } \approx \hbar \omega_{e}$, где $\hbar \omega_{e}$ - спектральное положение длинноволнового края интегрального по времени спектра излучения $w_{s}\left(\hbar \omega_{s}\right)$ (рис. 4). Длинноволновый склон спектра можно аппроксимировать экспонентой, а за $\hbar \omega_{e}$ принять энергию фотона, при которой энергия излучения $w_{s}$ начинает изменяться экспоненциально с $\hbar \omega_{s}$, что начинается приблизительно при $w_{s} \approx 0.79$ отн. ед. (см. вставку на рис. 4). Аналогичный метод определения края спектра $\hbar \omega_{e}$ использовался в $[21,25]$ и при подтверждении зависимости $E_{g}=f(n)$ в работе [7]. Полученные из эксперимента значения $\hbar \omega_{e}$ при разных $D$, представляющие, согласно вышесказанному, зависимость $E_{g-\min }(D)$, показаны на рис. 5 (кривая 1). Уже на этом этапе обращает на себя внимание сходство формы графиков экспериментально полученных зависимостей $E_{g}-\min (D)$ (рис. 5) и $\tau_{r}(D)$ (рис. 4). Такое сходство не противоречит выражению (10). Далее, для каждого $D$ находилось то значение $n$ на зависимости $E_{g}=f(n)$, которое соответствовало $E_{g}=\hbar \omega_{e}$ для этого $D$. Оно и принималось

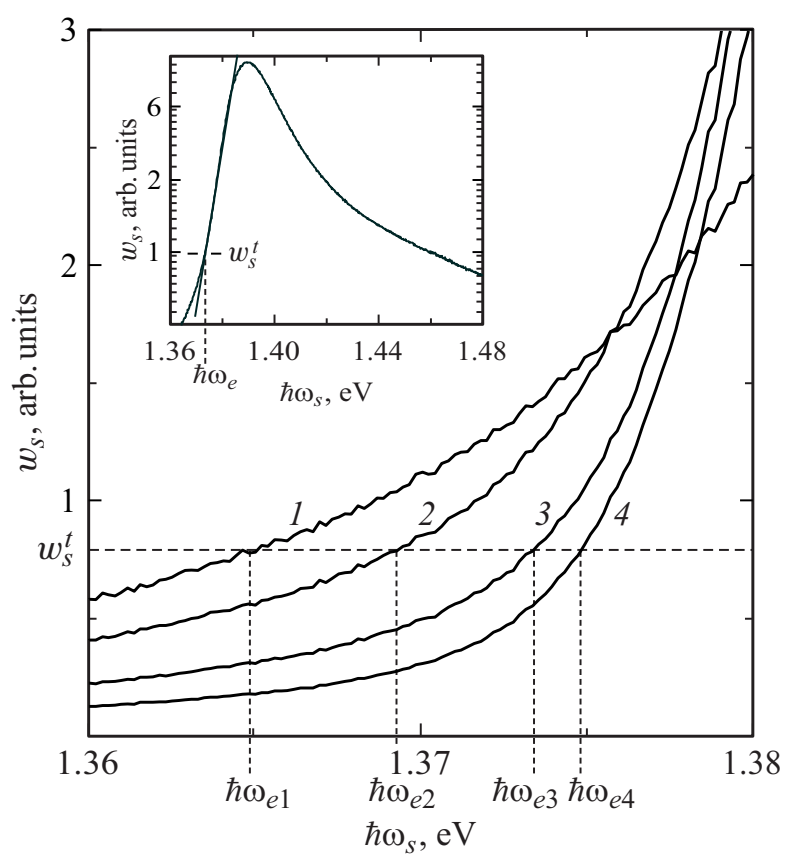

Рис. 4. Длинноволновый край интегрального по времени спектра излучения $w_{s}\left(\hbar \omega_{s}\right)$ при диаметрах $D$, мм: $1-0.21$, $2-0.54,3-0.7,4-0.82$. Пунктиром показан уровень плотности энергии излучения $w_{s}^{t}$, по которому определялась энергия фотона $\hbar \omega_{e}$, к которой приравнивалась предельно суженная ширина запрещенной зоны $E_{g-\min }$. Значения $\hbar \omega_{e}$, приведенные с индексом $i=1-4$ на оси абсцисс, относятся к спектрам 1-4 соответственно. На вставке - спектр излучения $w_{s}\left(\hbar \omega_{s}\right)$ при диаметре $D=0.7$ мм. К длинноволновому склону спектра проведена касательная, показывающая, что его можно аппроксимировать экспонентой. $\hbar \omega_{e}-$ энергия фотона, с которой энергия излучения $w_{s}$ начинает возрастать экспоненциально с $\hbar \omega_{s}$, что начинается при $w_{s}^{t} \approx 0.79$ отн. ед.

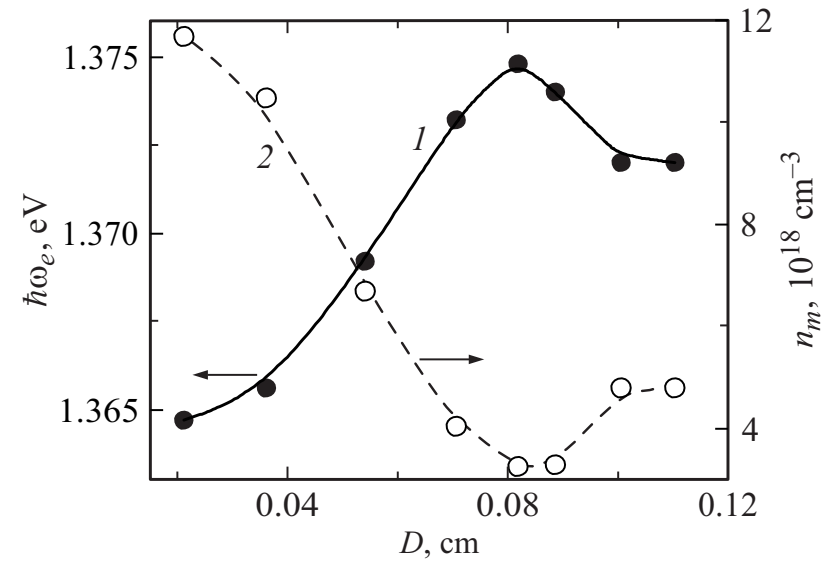

Рис. 5. Экспериментальные значения $\hbar \omega_{e}$ при разных диаметрах $D$, представляющие зависимость $E_{g-\min }(D)(1)$. Максимальная плотность ЭДП $n_{m}$ при разных диаметрах $D(2)$.

равным $n_{m}$. Плотность $n_{m}$ при разных $D$ представлена кривой 2 на рис. 5. Такой способ определения плотности носителей является предпочтительным по сравнению, например, с измерениями энергии импульса накачки. Энергия накачки не дает информации о плотности носителей заряда в GaAs, поскольку, во-первых, импульс накачки не полностью поглощается в образце. Во-вторых, во время накачки происходит интенсивная стимулированная рекомбинация носителей заряда. Как и в $[1,3]$, в формулу (10) будем подставлять параметры, соответствующие средней плотности электронов за время релаксации излучения $n_{a v}=\left(n_{m}+n_{\mathrm{th}}\right) / 2$. Соответствующие $n_{a v}$ значения температуры $T_{c}$, определялись из проведенной через экспериментальные точки зависимости $n\left(T_{c}\right)$, показанной пунктиром на рис. 6 в работе [3]. Подставляя полученные вышеописанным образом параметры в формулу (10), были рассчитаны значения $\tau_{r}$, представленные не закрашенными кружками на графике $\tau_{r}(D)$ (рис. 3). Видно, что экспериментальные и расчетные значения $\tau_{r}$ близки. Это согласие является доказательством связи времени остывания носителей заряда и релаксации излучения. Таким образом, используя формулу (10), удается получить правильную оценку времени $\tau_{r}$ и его зависимости от диаметра D. Заметим, что мы предпочли определять приблизительно среднее экспериментальное и среднее оценочное характерное время релаксация излучения. Однако в течение релаксации излучения взаимосвязанные параметры $E_{g}$, $T_{c}$ и $\tau_{p}$ меняются, поэтому в соответствии с (10) может меняться и $\tau_{r}$. Этого, в принятом в настоящей работе первом приближении, мы не учитывали.

Мы также пренебрегаем пространственным растеканием заряда за время излучения. Допустимость этого подтверждается следующим. В нашей статье [26] приведены для сравнения экспериментальные графики изменения в плоскости образца энергии и просветления. Последний график отображал одновременно и простран- 


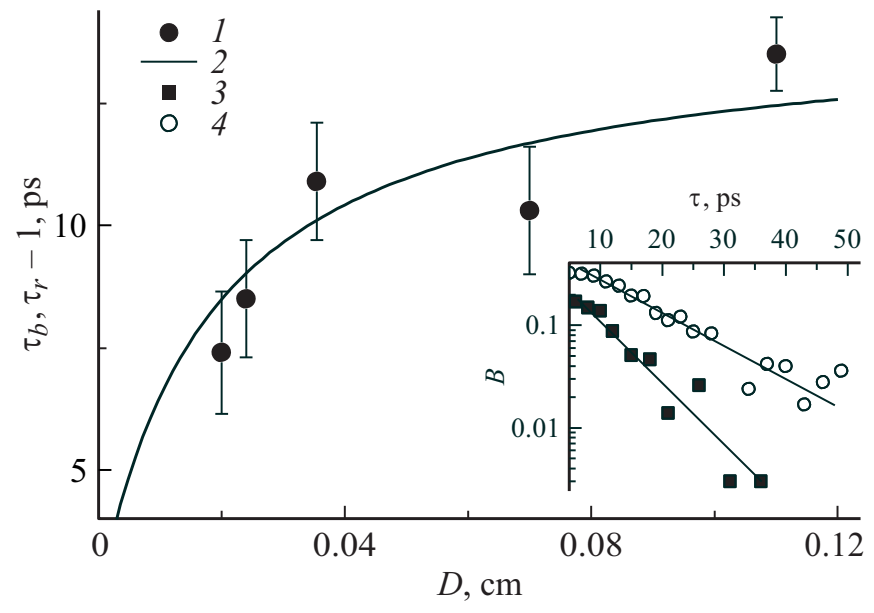

Рис. 6. Характерное время релаксации просветления $\tau_{b}$ при разных диаметрах $D(1)$. График расчетной зависимости характерного времени релаксации плотности носителей, опущенный на 1 пс: $\tau_{r}-1$ пс $=f(D)$ (2) (пояснения см. в тексте). На вставке - релаксация надпорогового вклада в просветление $B=\lg \left(T^{1} / T^{0}\right)-\lg \left(T^{1} / T^{0}\right)_{\text {th }}$ со временем задержки $\tau$ между возбуждающим и зондирующим импульсами (представлено в полулогарифмическом масштабе) при диаметрах $D$, см: $3-$ $0.02,4-0.11$.

ственное распределение неравновесных носителей. Графики, отнормированные на их амплитуду, практически не отличались. В работе [27] измеренный коэффициент пространственной диффузии носителей, накачиваемых фемтосекундными импульсами в $\mathrm{AlGaAs,} \mathrm{равнял-}$ ся $200 \mathrm{~cm}^{2} /$ с. Тогда за время 80 пс, равное максимальной длительности нашего излучения, диффузия в условиях работы [27] должна была бы произойти на 1.3 мкм. Эту оценку нельзя прямо относить к условиям наших опытов, но все же отметим, что эта длина на два с лишним порядка меньше минимального диаметра в наших опытах.

Обратимся теперь к работе [1], в которой анализировалась релаксация просветления $\lg \left(T^{1} / T^{0}\right)$ слоя GaAs до остаточного уровня $\lg \left(T^{1} / T^{0}\right)_{\text {th }}$, достигавшегося при пороговой плотности ЭДП $n_{\mathrm{th}}$, здесь $T^{1}$ и $T^{0}$ - прозрачность образца при наличии и в отсутствие накачки соответственно. Отмечалось, что изменение просветления отображало преимущественно изменение плотности ЭДП, а изменение температуры влияло на просветление лишь в очень незначительной степени, что подтверждает, например, рис. 11 [15]. Рассмотрим теперь релаксацию надпорогового вклада в просветление $B=\lg \left(T^{1} / T^{0}\right)-\lg \left(T^{1} / T^{0}\right)_{\mathrm{th}}=f(\tau)$ в [1]. Приблизительно экспоненциальная релаксация этого вклада иллюстрируется на вставке к рис. 6, а характерное время такой релаксации в функции от диаметра $D$ представлено на самом рисунке. На рисунке аппроксимирующая экспериментальные точки кривая 1 представляет график расчетной зависимости (10) для экспериментальных параметров $\tau_{p}, T_{c}, E_{g}$ в [1], опущенный на 1 пс. Последнее означает, что экспериментальное характерное время релаксации просветления $\tau_{b}$ слегка меньше расчетного $\tau_{r}$. Похоже, что эта незначительная разница вызвана неучтенным влиянием на изменение просветления понижения температуры. Отклонения экспериментальных точек от аппроксимирующей кривой связаны, по-видимому, с тем, что погрешность измерений в эксперименте [1] была больше, чем в настоящей работе, что объясняется различием методов измерений и менее совершенной аппаратурой. В целом же релаксация просветления, представленная графиками на рис. 6, позволяет считать, что релаксация надпороговой части плотности ЭДП $\Delta n=n-n_{\text {th }}$ не противоречит $(10)$.

\section{4. Заключение}

В итоге экспериментально обнаружено увеличение характерного пикосекундного времени $\tau_{r}$ релаксации излучения (и плотности носителей $n$ ), происходившее при увеличении диаметра импульса накачки. Плотность энергии импульса накачки была фиксированной. Предложенное аналитическое выражение для $\tau_{r}$ согласуется с экспериментальными результатами. Это позволяет использовать такое выражение на практике в условиях интенсивного стимулированного излучения GaAs (в состоянии насыщения усиления излучения). Аналитическое выражение для $\tau_{r}$ является функцией характерного времени релаксации температуры $\tau_{T}$ фотонакачанных носителей. Время $\tau_{T}$ было определено в [2] с учетом нагрева носителей, вызванного излучением.

\section{Благодарности}

Авторы глубоко признательны Ю.В. Андрееву за обсуждение рукописи статьи.

\section{Финансирование работы}

Работа выполнена в рамках государственного задания.

\section{Конфликт интересов}

Авторы заявляют, что у них нет конфликта интересов.

\section{Список литературы}

[1] И.Л. Броневой, А.Н. Кривоносов. ФТП, 32, 542 (1998).

[2] Ю.Д. Калафати, В.А. Кокин. ЖЭТФ, 99, 1793 (1991).

[3] Н.Н. Агеева, И.Л. Броневой, Д.Н. Забегаев, А.Н. Кривоносов. ЖЭТФ, 143, 634 (2013).

[4] Н.Н. Агеева, И.Л. Броневой, А.Н. Кривоносов, С.Е. Кумеков, С.В. Стеганцов. ФТП, 36, 144 (2002).

[5] N.N. Ageeva, I.L. Bronevoi, E.G. Dyadyushkin, V.A. Mironov, S.E. Kumekov, V.I. Perel'. Sol. St. Commun., 72, 625 (1989).

[6] Н.Н. Агеева, И.Л. Броневой, Е.Г. Дядюшкин, Б.С. Явич. Письма ЖЭТФ, 48, 252 (1988).

[7] Н.Н. Агеева, И.Л. Броневой, А.Н. Кривоносов. ФТП, 35, 65 (2001). 
[8] Х.-Г. Унгер. Планарные и волоконные оптические волноводы (М., Мир, 1980).

[9] Н.Н. Агеева, И.Л. Броневой, Д.Н. Забегаев, А.Н. Кривоносов. ЖЭТФ, 144, 227 (2013).

[10] Н.Н. Агеева, И.Л. Броневой, Д.Н. Забегаев, А.Н. Кривоносов. Радиотехника и электроника, 63, 1130 (2018).

[11] И.Л. Броневой, А.Н. Кривоносов. ФТП, 32, 537 (1998).

[12] Н.Н. Агеева, И.Л. Броневой, Д.Н. Забегаев, А.Н. Кривоносов. Журн. радиоэлектроники, 11, 1 (2018). http://jre.cplire.ru/jre/nov18/13/text.pdf

[13] J.S. Blakemore. J. Appl. Phys., 53, R123 (1982).

[14] М. Грундман. Основы бизики полупроводников (М., Физматлит, 2012).

[15] N.N. Ageeva, I.L. Bronevoi, S.E. Kumekov, V.A. Mironov, V.I. Perel'. [In: Mode-Locked Lasers and Ultrafast Phenomena, ed. by G.B. Altshuler] Proc. SPIE, 1842, 70 (1992).

[16] И.Л. Броневой, С.Е. Кумеков, В.И. Перель. Письма ЖЭТФ, 43, 368 (1986).

[17] С.Е. Кумеков, В.И. Перель. ЖЭТФ, 94, 346 (1988).

[18] N.N. Ageeva, I.L. Bronevoi, V.A. Mironov, S.E. Kumekov, V.I. Perel'. Sol. St. Commun., 81, 969 (1992).

[19] N.N. Ageeva, V.B. Borisov, I.L. Bronevoi, V.A. Mironov, S.E. Kumekov, V.I. Perel', B.S. Yavich. Sol. St. Commun., 75, 167 (1990).

[20] J. Shah, R.F. Leheny, C. Lin. Sol. St. Commun., 18, 1035 (1976).

[21] S. Tarucha, H. Kobayashi, Y. Horikoshi, H. Okamoto. Jpn. J. Appl. Phys., 23, 874 (1984).

[22] Т. Райс, Дж. Хенсел, Т. Филлипс, Г. Томас. Электроннодырочная жидкость в полупроводниках (М., Мир, 1980).

[23] Теория неоднородного электронного газа, под. ред. С. Лундквиста, Н. Марча (М., Мир, 1987).

[24] Н.Н. Агеева, И.Л. Броневой, Д.Н. Забегаев, А.Н. Кривоносов. ФТП, 51, 594 (2017).

[25] D. Olego, M. Cardona. Phys. Rev. B, 22, 886 (1980).

[26] Н.Н. Агеева, И.Л. Броневой, Д.Н. Забегаев, А.Н. Кривоносов. ЖЭТФ, 147, 765 (2015).

[27] K.V. Ivanin, A.V. Leontyev, V.S. Lobkov, V.V. Samartsev. Laser Phys. Lett., 7 (8), 583 (2010).

\section{Relation of relaxation of intrinsic stimulated picosecond emission of GaAs with the characteristic time of charge carriers cooling}

\author{
N.N. Ageeva, I.L. Bronevoi, D.N. Zabegaev, \\ A.N. Krivonosov \\ Kotel'nikov Institute of \\ Radioengeneering and Electronics, \\ Russian Academy of Sciences, \\ 125009 Moscow, Russia
}

Abstract During the powerful picosecond optical pumping of a thin $(\sim 1 \mu \mathrm{m})$ GaAs layer, a stimulated intense (up to $1 \mathrm{GW} / \mathrm{cm}^{2}$ ) picosecond emission appeared. As was found, for a fixed density of the pump pulse energy, with an increase of its diameter the characteristic picosecond time $\tau_{r}$ of the emission and carrier density $n$ relaxation increases. Due to interrelation of the density and the temperature of the carriers at high-intensity emission (in the saturation state of the emission amplification), time $\tau_{r}$ is associated with the characteristic temperature relaxation time $\tau_{T}$ of the photo-pumped carriers, which was determined earlier theoretically with the emission-caused carrier heating taken into account. The corresponding analytical expressions for $\tau_{r}$ as a functions of $\tau_{T}$ are consistent with the above experimental results.

Редактор Г.А. Оганесян 